\title{
Assessment of the pregnancy rates using sequential day 3 and day 5 embryo transfer in IVF/ ICSI patients
}

\author{
Priti Agrawal $^{1 *}$, Rishi Agrawal ${ }^{2}$, Anandi Lobo ${ }^{3}$
}

\begin{abstract}
${ }^{1}$ Department of Obstetrics, Gynecology and Infertility, ${ }^{2}$ Department of General and Laparoscopic surgery, ${ }^{3}$ Department of Pathology and IVF Unit Aarogya Hospital and Test Tube Baby Center, Raipur, Chhattisgarh, India
\end{abstract}

Received: 22 December 2020

Accepted: 01 February 2021

Accepted: 02 February 2021

\author{
*Correspondence: \\ Dr. Priti Agrawal, \\ E-mail: drpritiagrawal15@gmail.com
}

Copyright: (c) the author(s), publisher and licensee Medip Academy. This is an open-access article distributed under the terms of the Creative Commons Attribution Non-Commercial License, which permits unrestricted non-commercial use, distribution, and reproduction in any medium, provided the original work is properly cited.

\section{ABSTRACT}

Background: To assess the pregnancy rates using sequential day 3 and day 5 embryo transfer in IVF/ ICSI patients. Methods: This prospective study was conducted in Aarogya Hospital and test tube baby Centre, Raipur from $1^{\text {st }}$ January 2013 to $30^{\text {th }}$ November 2019. Total 100 patients undergoing IVF/ICSI in the study period were offered sequential transfer.

Results: Our fertilization rates were $80 \%$ with $85.7 \%$ grade I embryos on day 3. Blastocyst formation rate was $71.42 \%$. Cycle cancellation rates were nil. Clinical pregnancy rates per retrieval cycle were $50 \%$ and implantation rates were $24 \%$ with acceptable multiple pregnancy rates of $12 \%$.

Conclusions: We advocate that this technique is useful in all patients having good quality embryos in adequate number for double transfer as this optimizes the chance of selection of the most viable embryo for transfer which is probably the key for a successful IVF program.

Keywords: Blastocyst transfer, Embryo transfer, Repeated implantation failure, Sequential embryo transfer

\section{INTRODUCTION}

The clinical pregnancy rate following IVF is usually 30 $50 \%$ when there are no adverse maternal factors involving uterus, endometrium or immunological system, embryos are of good quality and adequate in number and maternal and paternal karyotypes are normal. The process of implantation involves two main components, a healthy embryo that should have the potential to implant and a receptive endometrium that should enable implantation. The "Cross-talk" between the embryo and the endometrium that finally leads to apposition, attachment and invasion of embryos is mandatory for successful implantation and subsequent normal placentation. ${ }^{1}$

The embryos obtained after IVF or ICSI are transferred into the uterine cavity either on day 2 or 3 (cleavage stage transfer) or day 5 or 6 (blastocyst transfer). Blastocyst transfer has inherent advantages compared to cleavage stage transfer such as better synchrony with the endometrium, lesser uterine contractility, better embryo euploidy status and higher implantation potential per embryo. Inadequate uterine receptivity is responsible for approximately two thirds of implantation failures, whereas the embryo itself is responsible for one third of these failures. In human's sequential embryo transfer may be used to increase endometrial receptivity and to have all advantages of blastocyst transfer.

Sequential transfer prevents the patient and the clinician of the major disadvantage in 'pure' blastocyst transfer of the cycle cancellation due to embryonic block in which there is a failure of embryos to proceed to blastocyst stage.

The objective of the study was to assess the pregnancy rates using sequential day 3 and day 5 embryo transfer in IVF/ ICSI patients. 


\section{METHODS}

This prospective study was conducted in Department of Obstetrics and Gynecology, Aarogya Hospital and test tube baby Centre from 1st January 2013 to 30th November 2019. Total 100 patients undergoing IVF/ ICSI were offered sequential transfer. Our inclusion criteria for the female partner was age $<37$ years, day 3 FSH level $<10$ IU/L, E2 $<80 \mathrm{pg} / \mathrm{ml}$, hysteroscopically normal endometrial cavity, all 10 follicles $>14 \mu \mathrm{m}$ in diameter on the day of receiving $\beta$ - HCG and 5 or more cleaved embryos on day 3 post fertilization. Thorough counseling of all the patients was done and informed consent was taken.

\section{Ovarian stimulation}

The standard gonadotrophin releasing hormone agonist long protocol (Mid luteal phase) was utilized. $0.5 \mathrm{ml}$ or 1 $\mathrm{mg}$ of leuprolide acetate was administered for down regulation from day 21 of preceding cycle and 100-300 IU of recombinant FSH was administered daily for ovarian stimulation. Follicle growth monitoring included serum estradiol, progesterone and LH measurements and transvaginal ultrasound. When one follicle reached a diameter $\geq 18 \mathrm{~mm}$ or two or more follicles reached $\geq 17$ $\mathrm{mm}$ and at least 10 follicles were more than $14 \mathrm{~mm}, 250$ $\mu \mathrm{g}$ of recombinant human chorionic gonadotrophin was administered and oocytes were retrieved 35-36 hours later. Routine IVF or ICSI was performed 4 hours after oocyte retrieval and the oocytes were checked for fertilization 16-18 hours later. Normal fertilization was indicated by appearance of two pronuclei. Embryos were cultured in commercial sequential IVF medium. Embryos were again observed at 48 hours (day 2) and 72 hours (day 3) after oocyte retrieval. The grading criteria for the embryos were as follows: Grade I, the size of the blastomeres was uniform, with no DNA fragmentation; Grade II, the blastomere size was slightly uneven with $<20 \%$ DNA fragmentation; Grade III, the blastomere size was heterogenous or with $20-50 \%$ DNA fragmentation and Grade IV, $>50 \%$ DNA fragmentation. The number and grade of the embryonic blastomeres were recorded. Good quality embryos were defined as embryos containing $\geq 4$ cells on day 2 and $\geq 6$ cells on day 3 with grade I-II.

In all the patients on day 3, two good quality embryos were transferred. Remaining embryos were placed in blastocyst culture medium and cultured until day 5. On day 5, one good quality blastocyst was transferred and the remaining blastocysts were frozen on the same day using vitrification technique. Blastocyst grading was done according to the Gardner and Schoolcraft grading system. $^{2}$ No embryo underwent assisted hatching before transfer. Luteal support consisted of $800 \mu \mathrm{g}$ of micronized progesterone orally initiated on the day of oocyte retrieval and continued until the day of pregnancy testing. Estrogen supplementation was given in the form of oral estradiol valerate $2 \mathrm{mg}$ tablets thrice a day.

\section{Outcome measures}

The primary outcome measures were the clinical pregnancy rate and implantation rate in the fresh cycle only. The secondary outcome measure was the miscarriage rate. Pregnancy testing was performed 14 days after embryo transfer. Ultrasound examination was performed at week 7 (about 5 weeks after transfer) to assess the fetal sac number and the fetal heartbeat. Clinical pregnancy was defined as the presents of a fetal heartbeat on ultrasound examination at 7 weeks of pregnancy. The implantation rate was defined as the number of gestational sacs seen on the ultrasound divided by the total number of embryos / blastocysts transferred. Implantation rate was calculated for all patients having ET and not just those who became pregnant. Spontaneous miscarriage was defined as a clinical pregnancy loss before 20 weeks of gestation age. Multiple pregnancies were defined as two or more gestational sacs observed on ultrasound. Frozen cycles were not taken into consideration and therefore cumulative pregnancy rates are not considered in this study.

The SPSS version 22.0 software program was used for statistical analysis. No ethical approval was needed for this study.

\section{RESULTS}

Our maximum number of patients $35 \%$ were in the age group of 27-32 years with mean age of 31 years, mean BMI was 24 and $75 \%$ patients had less than 10 years of infertility (Table 1).

Table 1: Patient profile.

\begin{tabular}{|lll|}
\hline $\mathbf{n}=\mathbf{1 0 0}$ & Number & Percentage \\
\hline Age (in years) & 30 & 30 \\
\hline $22-27$ & 35 & 35 \\
\hline $27-32$ & 18 & 18 \\
\hline $32-37$ & 10 & 10 \\
\hline $37-42$ & 7 & 7 \\
\hline$>42$ & & \\
\hline Mean age: 31 years & & 70 \\
\hline BMI & 70 & 30 \\
\hline $20-25$ & 30 & Nil \\
\hline $25-30$ & Nil & \\
\hline$>30$ & & \\
\hline Mean BMI:24 & 35 \\
\hline Duration of infertility (in years) & 40 \\
\hline$<5$ & 35 & 13 \\
\hline $5-10$ & 40 & 12 \\
\hline $10-15$ & 13 \\
\hline$>15$ & 12 \\
\hline Mean years of infertility: & years \\
\hline Type of infertility & \\
\hline Primary & 70 \\
\hline Secondary & 30 \\
\hline
\end{tabular}


Male Factors of infertility was predominant contributing to $59 \%$ cases (Table 2).

Table 2: Causes of infertility.

\begin{tabular}{|lll|}
\hline Factor & Number & Percentage \\
\hline Male factor & 59 & 59 \\
\hline Sperm parameters & & \\
\hline Azoospermia & 15 & 25.42 \\
\hline Oligospermia & 20 & 33.89 \\
\hline Oligoasthenoteratozoospermia & 15 & 25.42 \\
\hline Hypospermia & 05 & 8.47 \\
\hline Necrozoospermia & 02 & 3.38 \\
\hline Ejaculatory dysfunction & 02 & 3.38 \\
\hline Female factor & 32 & 32 \\
\hline Tubal factor & 10 & 31.25 \\
\hline Ovarian factor & 18 & 56.25 \\
\hline Uterine factor & 03 & 9.37 \\
\hline Cervical factor & 01 & 3.12 \\
\hline Combined factor & 20 & 20 \\
\hline Unexplained & 09 & 09 \\
\hline
\end{tabular}

Maximum $72 \%$ patients had antral follicle count (AFC) between $8-12$ and $90 \%$ patients had adequate endometrial thickness (ET) between 7-12 mm (Table 3).

Table 3: Relevant investigations.

\begin{tabular}{|lll|}
\hline $\mathbf{n = 1 0 0}$ & value & Number \\
\hline Hormonal assays (day 2) & & \\
\hline FSH (in ml IU/ml) & $<1$ & 6 \\
\hline & $1-4$ & 85 \\
\hline & $>4$ & 9 \\
\hline AMH (in ml IU/ml) & $<1$ & 10 \\
\hline & $1-4$ & 65 \\
\hline & $>4$ & 25 \\
\hline Serum E2 (day 5) (pg/ml) & $<500$ & 0 \\
\hline & $500-1000$ & 10 \\
\hline & $1000-1500$ & 65 \\
\hline & $1500-2000$ & 25 \\
\hline Antral folical count (day 2) & $4-8$ & 12 \\
\hline & $8-12$ & 72 \\
\hline & $>12$ & 16 \\
\hline $\begin{array}{l}\text { Endometrial thickness (on } \\
\text { day of OPU) }\end{array}$ & $<4 \mathrm{~mm}$ & 10 \\
\hline & $4-8 \mathrm{~mm}$ & 65 \\
\hline & $8-12 \mathrm{~mm}$ & 25 \\
\hline
\end{tabular}

A total 5-10 oocytes were retrieved in $65 \%$ cases (Table 4) with mean oocyte retrieval of 7 per patient, $90 \%$ oocytes were mature (M II). Our fertilization rates were $80 \%$ with $85.7 \%$ grade I embryos on day 3. Blastocyst formation rate was $71.42 \%$. Blastocyst were formed in all the patients and a single grade I blastocyst was transferred on day 5 to all patients. Surplus blastocyst was frozen for 25 patients. We had transferred 2 embryos on Day 3 and one blastocyst on Day 5 in all patients. Our clinical pregnancy rates per retrieval cycle were $50 \%$ but implantation rates were $24 \%$ (Table 5).

Table 4: Status of embryos.

\begin{tabular}{|lll|}
\hline $\mathbf{n}=\mathbf{1 0 0}$ & Patients & Percentage \\
\hline Number of oocytes & & \\
\hline Retrieved between & $\mathbf{6 5}$ & 65 \\
\hline $5-10$ & $\mathbf{2 5}$ & 25 \\
\hline $10-15$ & $\mathbf{1 0}$ & 10 \\
\hline$>15$ & $\mathbf{6 0 t a l} 700$ & oocytes) \\
\hline Mean oocyte per patient $=\mathbf{7}$ & To/700 & 90 \\
\hline M II oocytes & $560 / 700$ & $80 \%$ \\
\hline Oocytes fertilized & $480 / 560$ & 85.71 \\
\hline Grade I embryos on day 3 & $200 / 280$ & 71.42 \\
\hline Blastocyst formation rate & $140 / 280$ & 50.0 \\
\hline Grade I blastocyst on day 5 & & \\
\hline
\end{tabular}

Table 5: Outcome of sequential transfer.

\begin{tabular}{|lll|}
\hline $\mathbf{n = 1 0 0}$ & Number & Percentage \\
\hline $\begin{array}{l}\text { Clinical pregnancy rates (per } \\
\text { retrieval cycle) }\end{array}$ & 50 & 50 \\
\hline Implantation & $72 / 300^{*}$ & 24 \\
\hline Multiple pregnancy & 12 & 12 \\
\hline Early Pregnancy Loss & 10 & 10 \\
\hline Live pregnancy rate & 40 & 40 \\
\hline *2 day 3 embryo + 1 blastocyst transferred in all patients.
\end{tabular}

Multiple pregnancy occurred in $12 \%$ cases (Twins in all cases) and live pregnancy rates were $40 \%$.

\section{DISCUSSION}

There are various studies suggesting improved clinical pregnancy and implantation rates in patients with repeated IVF-ET failures. In studies by Ismail Madkour WA et al in sequential transfer group they had significantly higher pregnancy rate $(43.2 \%$ vs $27.4 \%)$, clinical pregnancy rate $(37.8 \%$ vs $21.9 \%)$, implantation rate $(17.1 \%$ vs $10.5 \%)$ and ongoing pregnancy rate (33.8\% vs $19.2 \%$ ) compared to conventional day 3 transfer. ${ }^{3}$ We offered double or sequential ET to all our patients having $\geq 5$ cleaved embryos on Day 3 even if it was their first IVF cycle. It is now suggested by various studies that to improve pregnancy rates endometrial receptivity should be increased. During first (day 3) transfer the embryos ( 2 embryos) in each patient may induce an increase in endometrial receptivity, thereby creating a better endometrial environment for the second transfer (1 blastocyst) on day 5 .

Also, co-culture of early stage embryos with endometrial epithelium may increase the success rate of IVF indicating that the interaction between the embryo and 
endometrium is important. Also, insertion of catheter during first transfer may induce mechanical stimulation of the endometrium and at the same time it also makes clinician aware of the technical difficulties which may occur during transfer like uterine manipulation or cervical grasping or even cervical dilatation and so these can be avoided during blastocyst transfer. Additionally, sequential transfer increases the chance of hitting the "implantation window" and thereby improving pregnancy rates. Goto el suggested that this method may be used not only in patients with repeated failures but also in poor prognosis patients even in their first IVF-ET in order to improve pregnancy rate. ${ }^{4}$

We got twin pregnancies in $12 \%$ of our patient Dalal et al found multiple pregnancy rate of $15.3 \%$ in the sequential transfer group. ${ }^{5}$ Alone blastocyst transfer on day 5 can have high cancelation rates because if no blastocyst is formed than no embryo can be transferred. Cycle cancelation rates were nil in our study. Fang et al also reported no cycle cancelations in day 3 and day 5 transfer group. ${ }^{6}$

With sequential transfer we could achieve clinical pregnancy rate of $50 \%$ which is comparable with Loutradis D et al who reported $60 \%$ clinical pregnancy rate with an additional embryo transfer on day 4.7 Tehraninejad et al found that chemical and clinical pregnancy rate was similar in the sequential ET GROUP (40\%) compared to the day 5 of ET group (38.3\%) I patients with three repeated IVF-ET failures. ${ }^{8}$

We would like to emphasis that surplus embryos add additional cost of freezing of embryos. If sequential transfer is done patient gets the best embryos and then surplus blastocyst can be frozen. We used vitrification for surplus blastocysts as the available data supports its potential safety. ${ }^{9}$

Limitation of the study: We had limited number of case who agreed for sequential transfer considering the fact that may couples refused to enroll in the study due to the risk of multiple gestation and wanted single embryo transfer only.

\section{CONCLUSION}

Our experience with sequential transfer has improved our clinical pregnancy rates from $30 \%$ to $50 \%$. We have not faced the problems of cycle cancelation. All the good quality embryos are properly utilized. It is also reduced our cost of Day 3 embryo freezing. Our experience with blastocyst freezing and its outcome still needs long term follow-up. We advocate that this technique is useful in all patients having good quality embryos in adequate number for double transfer as this optimizes the chance of selection of most viable embryo for transfer which is probably the key for a successful IVF program.

Funding: No funding sources

Conflict of interest: None declared

Ethical approval: The study was approved by the Institutional Ethics Committee

\section{REFERENCES}

1. Simon A, Laufer N: Assessment and treatment of repeated implantation failure (RIF); J Assist Reprod Genet. 2012;29:1227-39.

2. Gardner DK, School Craft WB, Wagley L, Schlenker T, Stevens J, Hesla J. A prospective randomized trial of blastocyst culture and transfer in in-vitro fertilization, Human Reprod. 1998;13:3434-40.

3. Ismail Madkour WA, Noah B, Zaheer H, Bahr A A, Abdelhamid A M.S, Shaeer M et al: Does sequential embryo transfer improve pregnancy rate in patients with repeated implantation failure? A randomized control studies. J Middle East Fertility Society 2015.

4. Goto S, Takcbayashi K, Shiotani M, Fuji Wara M, Hirose M, Noda Y. Effectiveness of 2 step consecutive transfer J Repord Med. 2003;48:370-4.

5. Dalal R, Mishra A, Pai HD, Palshetkar N. A prospective trial comparing sequential day $3 /$ day 5 transfer with cleavage stage transfer and blastocyst stage transfer. J Minimal Stimulation IVF. 2015; 2:30-6.

6. Fang C, Huang R, Li TT, Jia L, Li LL, Liang XY. Day -2 and day -3 sequential transfer improves pregnancy rate in patients with repeated IVF embryo transfer failure: a retrospective case - control study. Reproductive Bio Medicine online. 2013;26: 30-5.

7. Loutraids D, Drakakis P, Dallianidis K, Bletsa SR, Millingos S, Doumplis N, et al. A double embryo transfer on day 2 and 4 or 5 improves pregnancy outcome In patients with good embryos but repeated failures in IVF or ICSI. J Clin Exp Obstet Gynecol. 2004;31(1):63-6.

8. Tehraninejad E S, Raisi E, Ghaleh F B, Rashidi B H, Aziminekoo E, Kalantari V, Haghollahi F, Shariat $\mathrm{M}$, The sequential embryo transfer compared to blastocyst embryo transfer in in vitro fertilization (IVF) cycle in patients with the three repeated consecutive IVF. A randomized controlled trial, Gynecol Endocrinol. 2019;35(11):955-9.

9. Kader AA, Choi A, Orief Y, Agrawal A. Factors affecting the outcome of human blastocyst vitrification. J Reproductive Biology and Endocrinol. 2009;7:99:1-11.

Cite this article as: Agrawal P, Agrawal R, Lobo A. Assessment of the pregnancy rates using sequential day 3 and day 5 embryo transfer in IVF/ ICSI patients. Int J Reprod Contracept Obstet Gynecol 2021; 10:1042-5. 